\title{
Acetyl-L-Carnitine Induces Autophagy to Promote Mouse Spermatogonia Cell Recovery after Heat Stress Damage
}

\author{
Na Qiao $\left(D,{ }^{1}\right.$ Hanming Chen, ${ }^{1}$ Peiquan Du, ${ }^{1}$ Zhenlong Kang, ${ }^{1}$ Congying Pang, ${ }^{1}$ Bingxian Liu, ${ }^{1}$ \\ Qiwen Zeng, ${ }^{1}$ Jiaqiang Pan, ${ }^{1}$ Hui Zhang, ${ }^{1}$ Khalid Mehmood $\mathbb{D}^{2},{ }^{2}$ Zhaoxin Tang ${ }^{D}$, \\ and Ying $\mathrm{Li}$ (1) \\ ${ }^{1}$ College of Veterinary Medicine, South China Agricultural University, Guangzhou 510642, China \\ ${ }^{2}$ Faculty of Veterinary \& Animal Sciences, The Islamia University of Bahawalpur, Bahawalpur 63100, Pakistan
}

Correspondence should be addressed to Zhaoxin Tang; tangzx@scau.edu.cn and Ying Li; lying@scau.edu.cn

Received 8 September 2020; Revised 20 December 2020; Accepted 4 January 2021; Published 19 January 2021

Academic Editor: A. Ortega

Copyright ( $2021 \mathrm{Na}$ Qiao et al. This is an open access article distributed under the Creative Commons Attribution License, which permits unrestricted use, distribution, and reproduction in any medium, provided the original work is properly cited.

\begin{abstract}
Acetyl-L-carnitine (ALC) is an effective substrate for mitochondrial energy metabolism and is known to prevent neurodegeneration and attenuate heavy metal-induced injury. In this study, we investigated the function of ALC in the recovery of mouse spermatogonia cells (GC-1 cells) after heat stress (HS). The cells were randomly divided into three groups: control group, HS group (incubated at $42^{\circ} \mathrm{C}$ for $90 \mathrm{~min}$ ), and $\mathrm{HS}+\mathrm{ALC}$ group (treatment of $150 \mu \mathrm{M}$ ALC after incubated at $42^{\circ} \mathrm{C}$ for $90 \mathrm{~min}$ ). After heat stress, all of the cells were recovered at $37^{\circ} \mathrm{C}$ for $6 \mathrm{~h}$. In this study, the content of intracellular lactate dehydrogenase (LDH) in the cell supernatant and the malondialdehyde (MDA) levels, catalase (CAT) levels, and total antioxidant capacity (T-AOC) were significantly increased in the HS group compared to the CON group. In addition, the mitochondrial membrane potential (MMP) was markedly decreased, while the apoptosis rate and the expression of apoptosis-related genes (Bcl-2, Bax, and caspase3) were significantly increased in the HS group compared to the CON group. Furthermore, the number of autophagosomes and the expression of autophagy-related genes (Atg5, Beclin1, and LC3II) and protein levels of p62 were increased, but the expression of LAMP1 was decreased in the HS group compared to the CON group. However, treatment with ALC remarkably improved cell survival and decreased cell oxidative stress. It was unexpected that levels of autophagy were markedly increased in the HS + ALC group compared to the HS group. Taken together, our present study evidenced that ALC could alleviate oxidative stress and improve the level of autophagy to accelerate the recovery of GC-1 cells after heat stress.
\end{abstract}

\section{Introduction}

Global warming is causing more and more organisms to be under heat stress which influences animal growth, development, and reproduction $[1,2]$. The male reproduction is one of the most sensitive processes to be damaged by heat stress because the optimal temperature for spermatogenesis is slightly lower than that of the body [3,4]. Among the germ cells, the pachytene spermatocytes and the sperm are very vulnerable to heat [5-7]. Some studies have correlated that heat stress can affect spermatogenesis to fail by increasing the apoptosis of testicular germ cells, and it can also lead to the fragmentation of sperm DNA $[8,9]$. In marked contrast, spermatogonia appear relatively resilient to heat stress [10]. Heat-resistant spermatogonia can gradually recover and differentiate into new sperm after heat stress, which can gradually improve sperm quality and reduce the germ cell apoptosis [7]. So the resilience of spermatogonia plays a vital role in the recovery of sperm quality after heat stress. However, few studies have focused on the recovery capability of spermatogonia after heat stress.

Autophagy is a cytoprotective mechanism for cells to gain energy by phagocytosing organelles and proteins under various stress [11-13]. The function of autophagy in cell survival is complex. On the one hand, autophagy can degrade cellular components to maintain cell survival under stress. On the other hand, excessive autophagy has also been shown to promote cell apoptosis under certain conditions. A previous study has reported that heat stress increased autophagy in the testes of mice [14]. Furthermore, oxidative stress is considered 
to be one of the leading causes of damages of germ cells in heat stress [9]. Nonetheless, when spermatogonia are exposed to heat, the underlying mechanisms of autophagy remain unclear.

Acetyl-L-carnitine (ALC) is a natural substance found in the body. It is an effective substrate for mitochondrial energy metabolism and plays a crucial role in the oxidation of fatty acid $[15,16]$. ALC can prevent neurodegeneration and attenuate heavy metal-induced injury through improve mitochondrial functions and antioxidant capacity [17-19]. In addition, many clinical studies have shown that oral ALC increases the motility of spermatozoa, the velocity of spermatozoa, and the ratio of living spermatozoa [20, 21]. However, the protective effect of ALC on GC-1 cells after heat treatment remains unclear. In this study, we explored the ability of ALC to promote mouse spermatogonia cell recovery after heat stress damage and demonstrated its ability to antioxidant functions and upregulation of autophagy.

\section{Materials and Methods}

2.1. Cell Line and Reagents. The mouse-derived spermatogonia cell line (GC-1 cell line) was bought from American Type Culture Collection (ATCC) and maintained in DMEM medium (Gibco, NY, USA) supplemented with 10\% FBS (Gibco, NY, USA). Primary antibodies, anti-Beclin1 and anti-LC3, were from Sigma-Aldrich; anti-GAPDH was from Bioss Biotechnology. Secondary antibodies for immunoblot analysis were from Bioss Biotechnology. ALC was from Santa Cruz Biotechnology. Cell counting kit-8 (CCK-8) solution was from Dojindo Molecular Technologies Institute. Catalase (CAT) (A007-1-1), malondialdehyde (MDA) (A003-1-2), and total antioxidant capacity (T-AOC) (A015-1-2) assay kits were from Nanjing Jiancheng Bioengineering Institute (http://www.njjcbio.com/product .asp?cid=27\&sort=saleorder). $\quad 5,5^{\prime}, 6,6^{\prime}$-Tetrachloro-1, $1^{\prime}, 3,3^{\prime}$ -tetraethylbenzimidazolyl-carbocyanine iodide (JC-1), monodansylcadaverine (MDC), 4',6-diamidino-2-phenylindole (DAPI), and acridine orange/ethidium bromide (AO/EB) staining Kit were from Beyotime Biotechnology (https://www .beyotime.com/index.htm). Trizol reagent and Prime Script reverse transcriptase reagent kit were from TaKaRa Biotechnology.

2.2. Cell Culture and Heat-Shock Treatment. Cells were seeded and divided into three treatment groups: CON group, cells without any treatment; HS group, cells treatment with heat stress for $90 \mathrm{~min}$; and HS-ALC group, cells treated with $150 \mu \mathrm{M}$ ALC after heat stress. The CON group was placed into an incubator at $37^{\circ} \mathrm{C}$, whereas the experimental groups were incubated at $42^{\circ} \mathrm{C}$ incubator for heat stress for $90 \mathrm{~min}$. ALC was dissolved in DMEM to yield a $150 \mathrm{mM}$ stock solution and was dissolved in culture medium to yield the working solution with $150 \mu M$ ALC, while the same volume of DMEM was added to the control and the HS groups. After heat stress, all of the cells were recovered in a $37^{\circ} \mathrm{C}$ incubator for $6 \mathrm{~h}$.

2.3. Cell Viability Assay. GC-1 cells were seeded in 96-well plates at a density of 5000 cells per well. Then, $10 \mu \mathrm{L}$ CCK8 solution was added to each well after cells were treated according to the experiment. After cells were maintained at $37^{\circ} \mathrm{C}$ for $1 \mathrm{~h}, \mathrm{OD}$ values were measured at $450 \mathrm{~nm}$ using microplate spectrophotometer.

2.4. Lactate Dehydrogenase (LDH) Assay. The LDH content in cell growth medium at $6 \mathrm{~h}$ after heat stress treatment was assayed using an automatic biochemical analyzer (BS-380; Mindray, Shenzhen, China).

2.5. Determination of Oxidative Stress Indicators. Firstly, $0.25 \%$ trypsin was used to digest the cells, collecting $10^{7}$ cells. Then, $1 \mathrm{~mL}$ of normal saline was assed to the cells, and the cells were broken with homogenizer. After further centrifugation at $1000 \times \mathrm{g}$ for $15 \mathrm{~min}$, the supernatant is collected to determine CAT activity, MDA levels, and T-AOC activity. All experimental procedures were performed according to the reagent instructions.

2.6. Mitochondria Membrane Potential (MMP) Assay. MMP was evaluated by using the JC-1 solution according to the manufacturer's instruction. In brief, cells were collected and incubated with JC-1 staining solution inside the $\mathrm{CO}_{2}$ incubator for $30 \mathrm{~min}$, then rinsed and resuspended in JC-1 buffer. Finally, quantification by flow cytometry (CytoFLEX; Beckmen) detected mitochondria containing red JC-1 aggregates in PE (excitation: $490 \mathrm{~nm}$; emission: $530 \mathrm{~nm}$ ) channel and green JC-1 monomers in the FITC channel (excitation: $525 \mathrm{~nm}$; emission: $590 \mathrm{~nm}$ ).

2.7. Quantitative PCR Analysis. Quantitative PCR analysis was performed according to a method described previously [22]. In briefly, the cells were harvested in Trizol reagent for total RNA extraction. Prime Script reverse transcriptase reagent kit was used to reverse transcribe total RNAs into complementary DNA. PCRs were set up using a Detection System (Light Cycler 480II; Roche, USA). Relative mRNA levels of target genes were analyzed by the $2^{-\triangle \triangle \mathrm{Ct}}$ method. GAPDH was used as the reference gene. Specific primer sequences are listed in Table 1.

2.8. Monodansylcadaverine (MDC) Staining. GC-1 cells were harvested and incubated with $\mathrm{MDC}$ at $37^{\circ} \mathrm{C}$ for $30 \mathrm{~min}$. Then, cells were washed with PBS. Finally, the image of the cells was conducted under a fluorescence microscope (DM i8; Lecia, Germany) and observed at $512 \mathrm{~nm}$ when excited at $355 \mathrm{~nm}$.

2.9. Western Blot Analysis. Total proteins were homogenized in RIPA lysis buffer containing $1 \mathrm{mM}$ phenylmethanesulfonyl fluoride (PMSF). Then, proteins were quantified by the BCA assay kit. Equal proteins $(10 \mu \mathrm{g})$ of each sample were loaded onto SDS-PAGE gels. The blot was transferred onto polyvinylidene difluoride (PVDF) membranes and blocked with $5 \%$ nonfat milk for $1 \mathrm{~h}$ at room temperature and incubated with primary antibodies for $16 \mathrm{~h}$ at $4^{\circ} \mathrm{C}$. Following the incubation of the secondary antibody, the blot was developed by the electrochemiluminescence liquid (ECL) method by using the Image Lab software (Biorad, CA, USA). The density of the blot was analyzed by Image J.

2.10. Immunofluorescence Assay. GC-1 cells were fixed in 4\% paraformaldehyde and blocked with 5\% BSA. Cells then were 
TABLE 1: Specific primers used for real-time PCR.

\begin{tabular}{lcc}
\hline Gene name & Forward primer sequences $\left(5^{\prime} \rightarrow 3^{\prime}\right)$ & Reverse primer sequences $\left(5^{\prime} \rightarrow 3^{\prime}\right)$ \\
\hline GAPDH & CCACCAACTGCTTAGCCCCC & ATTCCTGGACCCAAAACGCT \\
Atg5 & ACAAGCAGCTCTGGATGGGACT & CCGCTCCGTCGTGGTCTGATAT \\
Beclin1 & TGATCCAGGAGCTGGAAGAT & CAAGCGACCCAGTCTGAAAT \\
LC3II & TGTCCACTCCCATCTCCGAAGT & TTGCTGTCCCGAATGTCTCCTG \\
Bax & TCCACCAAGAAGCTGAGCGAGT & CAGGGCCTTGAGCACCAGTTTG \\
Bcl-2 & ACCGTCGTGACTTCGCAGAGAT & TCTCCCTGTTGACGCTCTCCAC \\
Caspase3 & TGGAGGCTGACTTCCTGTATGC & ATTCCGTTGCCACCTTCCTGTT \\
Caspase9 & TCCTGGTACATCGAGACCTTG & AAGTCCCTTTCGCAGAAACAG \\
Mcoln1 & TGGTGCTGAGCCTCTTCATTGC & ACTGCCACGACGGAACTTGC \\
Clcn7 & AGGCGAGAGAAGGTTGGCATCA & GCTGGCTGGGTGTCTCCTACAT \\
Lamp1 & CGAGTGGCAACTTCAGCAAGGA & CAGCAGGCAGGTTCCGTTGTT \\
Lamp2 & GCAGTACCTGACAAGGCGACAC & CACAGCCCAAGAGACAGCGAAT \\
Ctsb & GCAGGCTGGACGCAACTTCTAC & TCACCGAACGCAACCCTTCCT \\
Ctsd & CGGCGTCTTGCTGCTCATTCT & ACACTGGCTCCGTGGTCTTAG \\
\hline
\end{tabular}

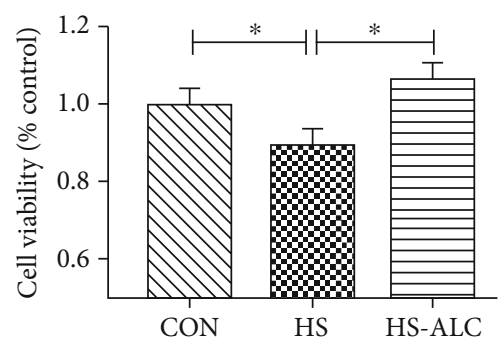

(a)

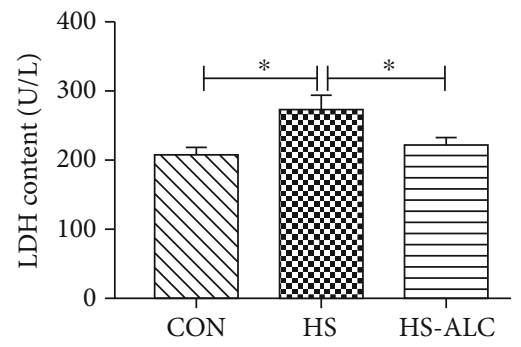

(b)

FIGURE 1: Effect of heat stress and ALC on cell viability and LDH release: (a) cell viability of GC-1 cells detected by CCK-8; (b) LDH content in supernatants. The data are presented as the mean $\pm \mathrm{SD}, n=3,{ }^{*} P<0.05,{ }^{* *} P<0.01$. CON: control group; HS: recover at 6 h; HS-ALC: recover at $6 \mathrm{~h}$ with ALC.

incubated with rabbit anti-LC3II antibody $(1: 200)$ for $16 \mathrm{~h}$ at $4^{\circ} \mathrm{C}$ and washed with PBS. Secondary antibodies conjugated to FITC were used. Cell nuclei were stained using DAPI. The image was taken by a Leica SP8 confocal microscope (Leica, Germany). Finally, the photographs were analyzed by ImageJ.

2.11. Apoptosis Analysis. After cells were treated according to the experiment, cells were harvested and resuspended in PBS. Then, cells were stained using the AO/EB staining Kit according to the instructions. The cells were scanned at emission/excitation wavelengths of $488 / 512 \mathrm{~nm}$ and $510 / 595 \mathrm{~nm}$, respectively. Finally, cells were analyzed with a fluorescent microscope (DM i8; Lecia, Germany).

2.12. Statistical Analysis. Data were analyzed using SPSS 18.0. Student's $t$-test or one-way analysis of variance (ANOVA) was used to analyzed the $P$ value $(* P<0.05 ; * * P<0.01)$. Data in this study were expressed as the mean \pm standard deviation (SD). Graphs are plotted using software GraphPad Prism 6.

\section{Results}

3.1. Effect of ALC on Cell Proliferation and LDH Release in GC-1 Cells after Heat Stress. Results of CCK-8 showed that cell viability was markedly reduced in the HS group $(P<0.05)$ compared to the CON group. However, the decrease of cell viability induced by heat stress was obviously reversed by ALC $(P<0.05)$ (Figure $1(\mathrm{a}))$. Besides the cell viability, GC-1 cell injuries were also measured by determining $\mathrm{LDH}$ activities in the media after $12 \mathrm{~h}$ culture. $\mathrm{LDH}$ leakage from GC-1 cells was significantly elevated in the HS group compared to the CON group $(P<0.05)$ (Figure $1(\mathrm{~b}))$, but this increase was remarkedly reduced after the combined administration of ALC $(P<0.05)$ (Figure $1(b))$. In addition, no obvious differences in LDH activities were observed between the CON group and the HS-ALC group $(P>0.05)$ (Figure 1(b)).

3.2. Oxidative Damage and Antioxidant Ability in GC-1. The activities of CAT and T-AOC and the concentration of MDA were measured to investigate the oxidative damage and 


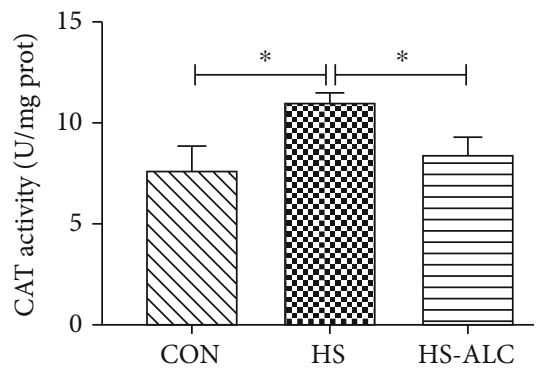

(a)

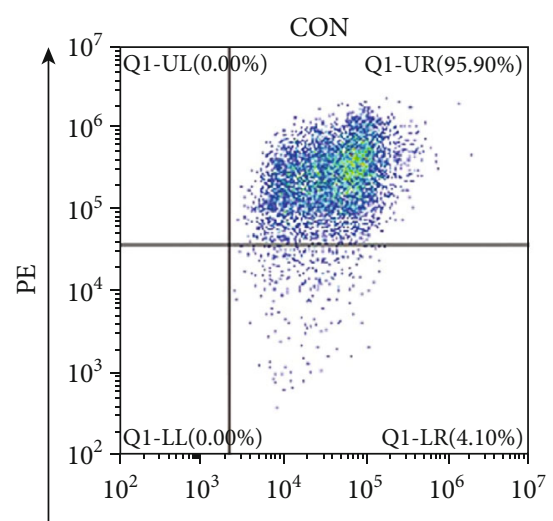

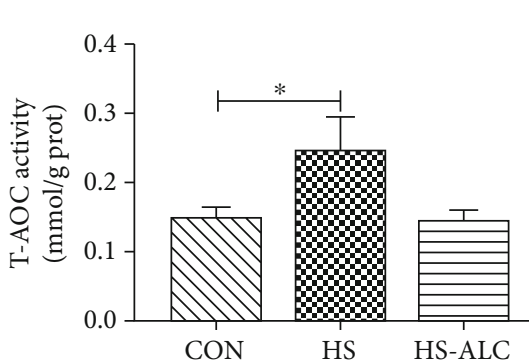

(b)

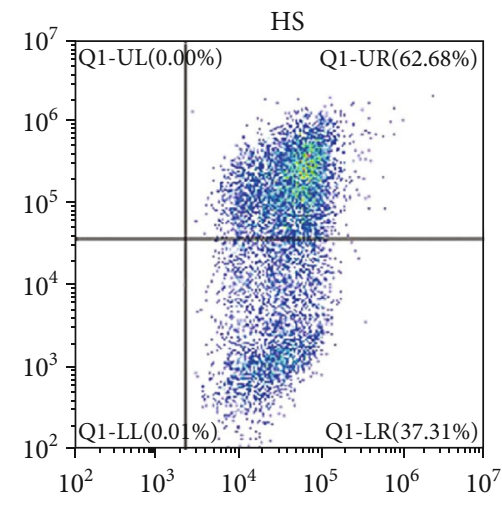

FITC

(d)

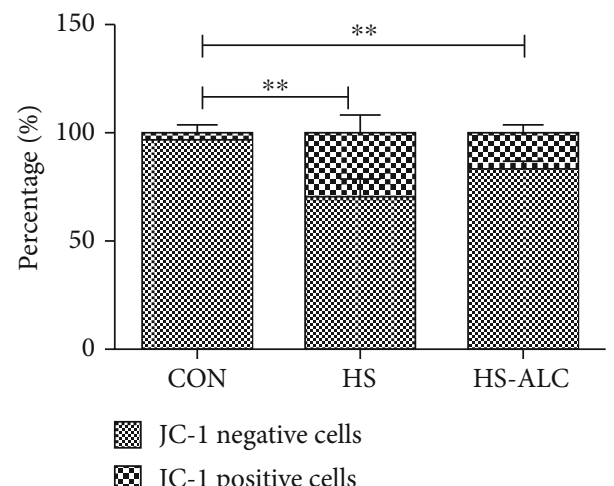

(e)

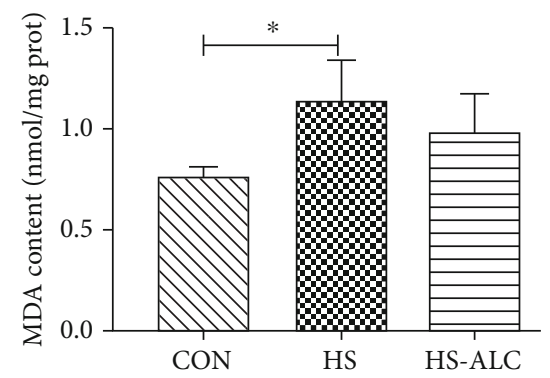

(c)

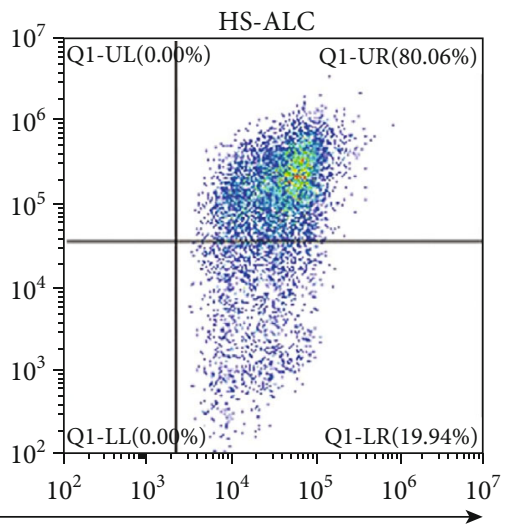

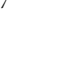



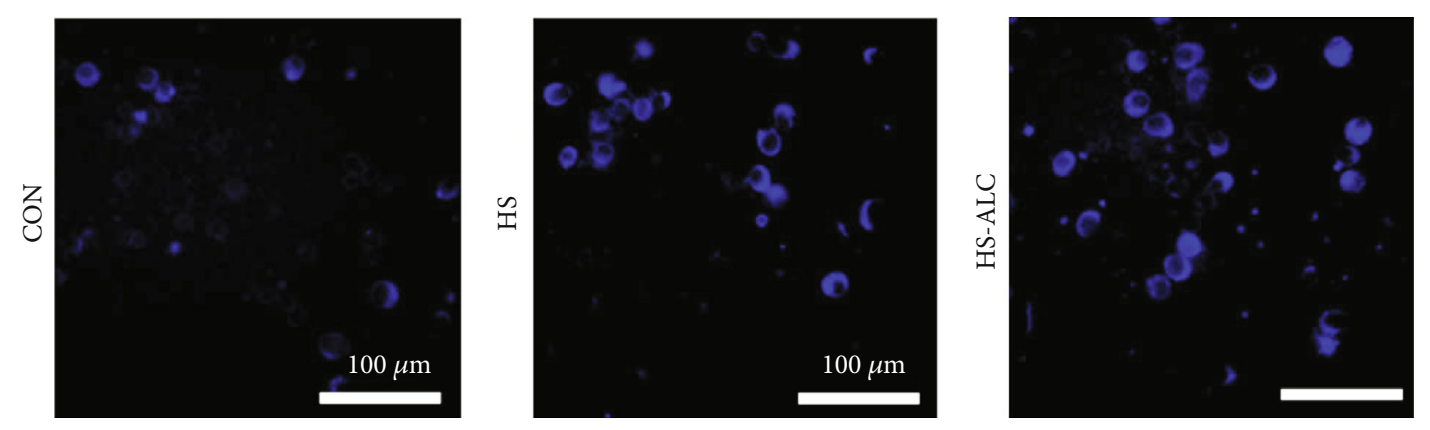

(a)
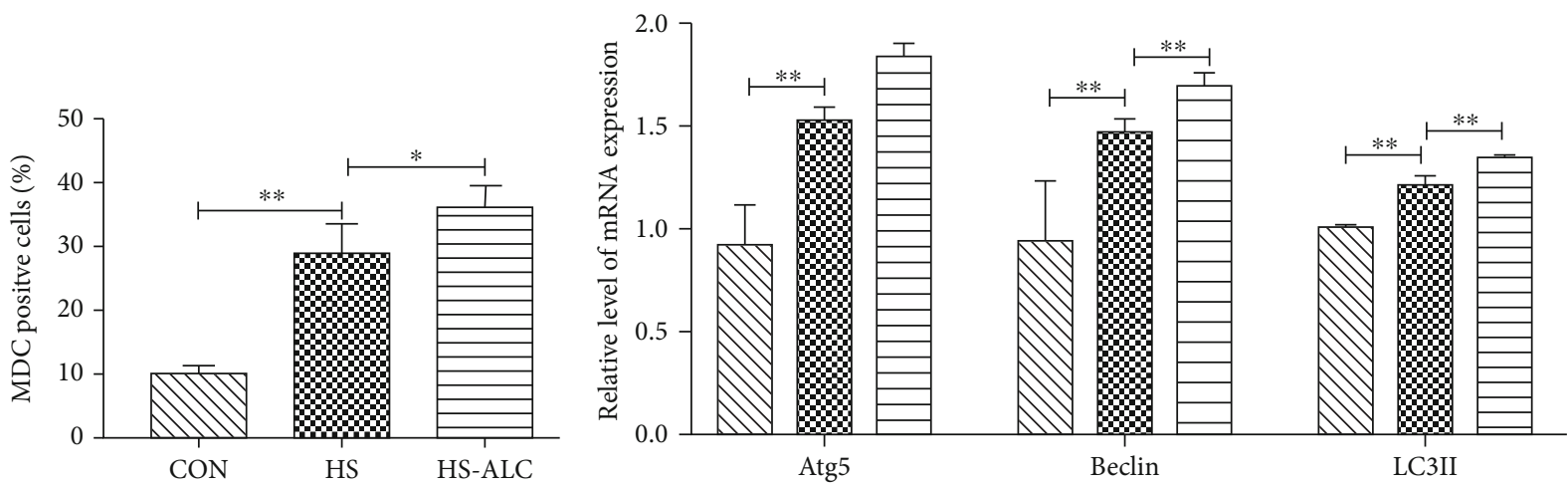

$\triangle \mathrm{CON}$

$8 \mathrm{HS}$

目 HS-ALC

(b)

(c)
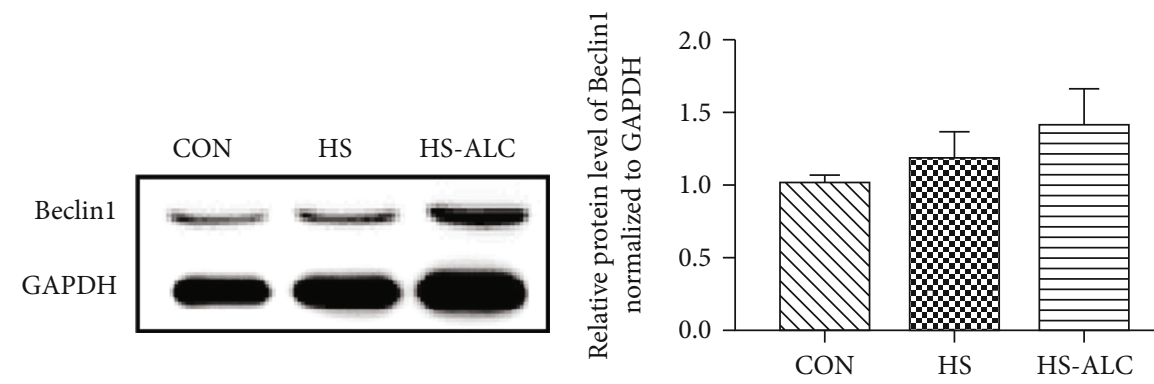

(d)

(e)
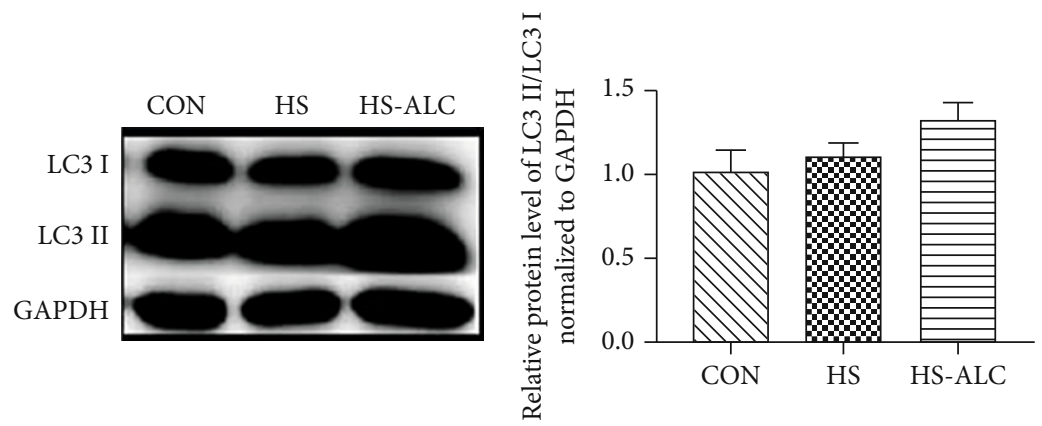

(f)

(g)

FIgURe 3: Continued. 

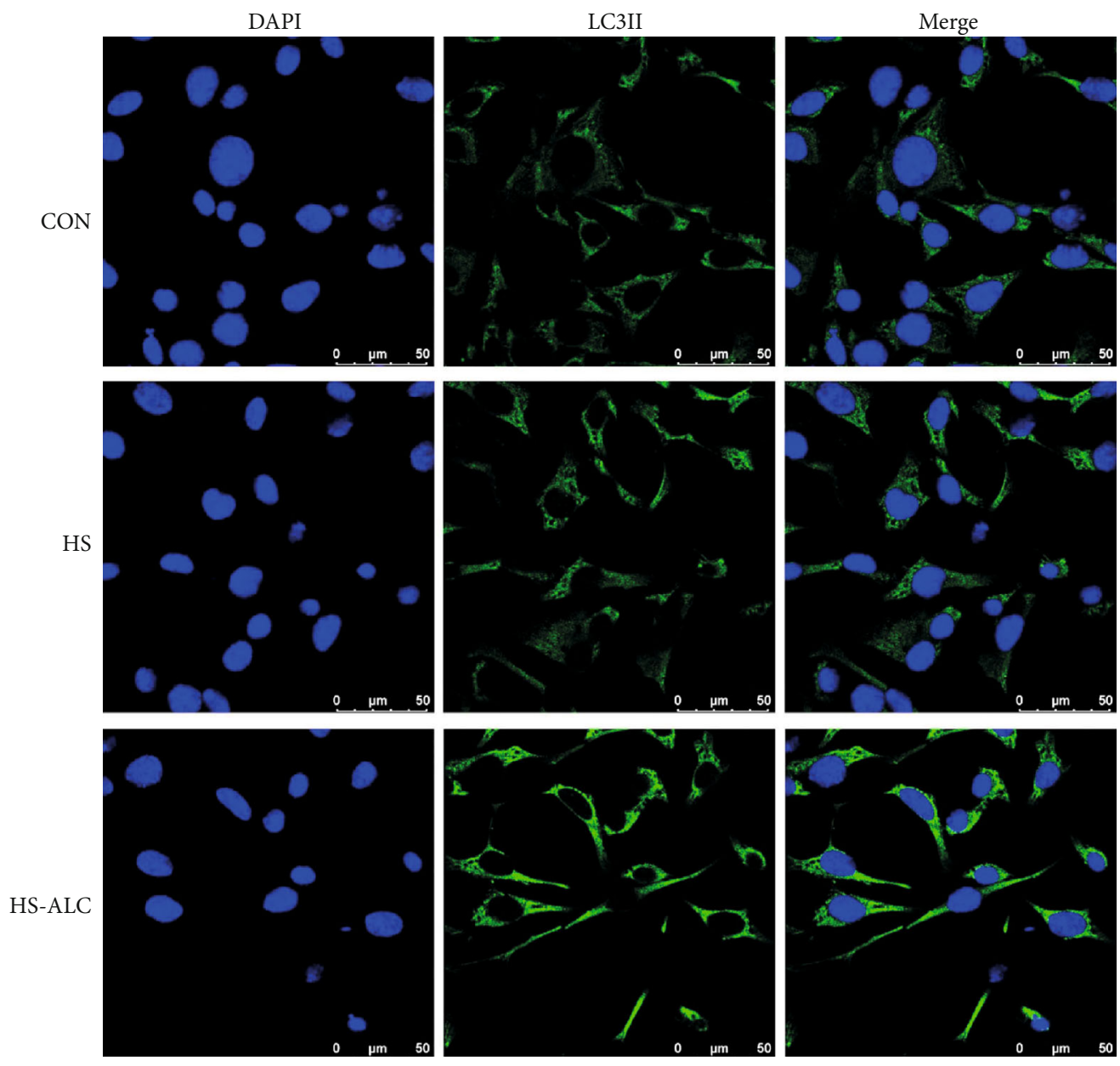

(h)

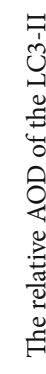

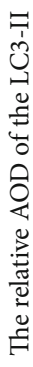

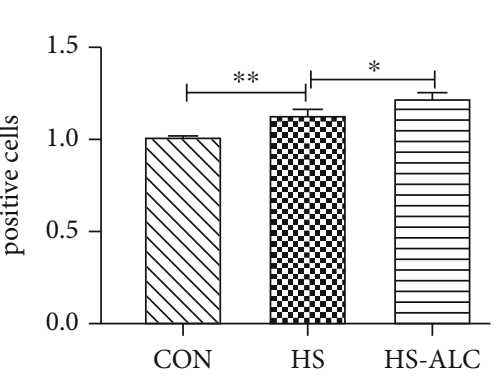

(i)

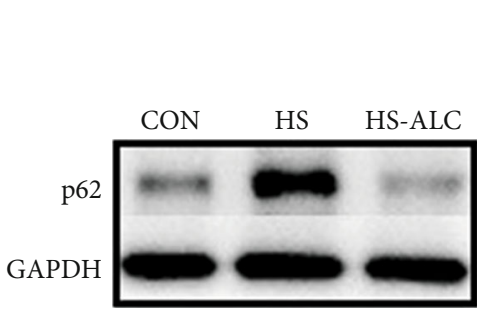

(j)

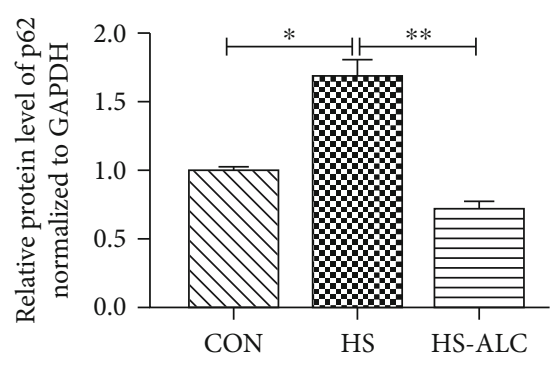

(k)

Figure 3: Continued. 


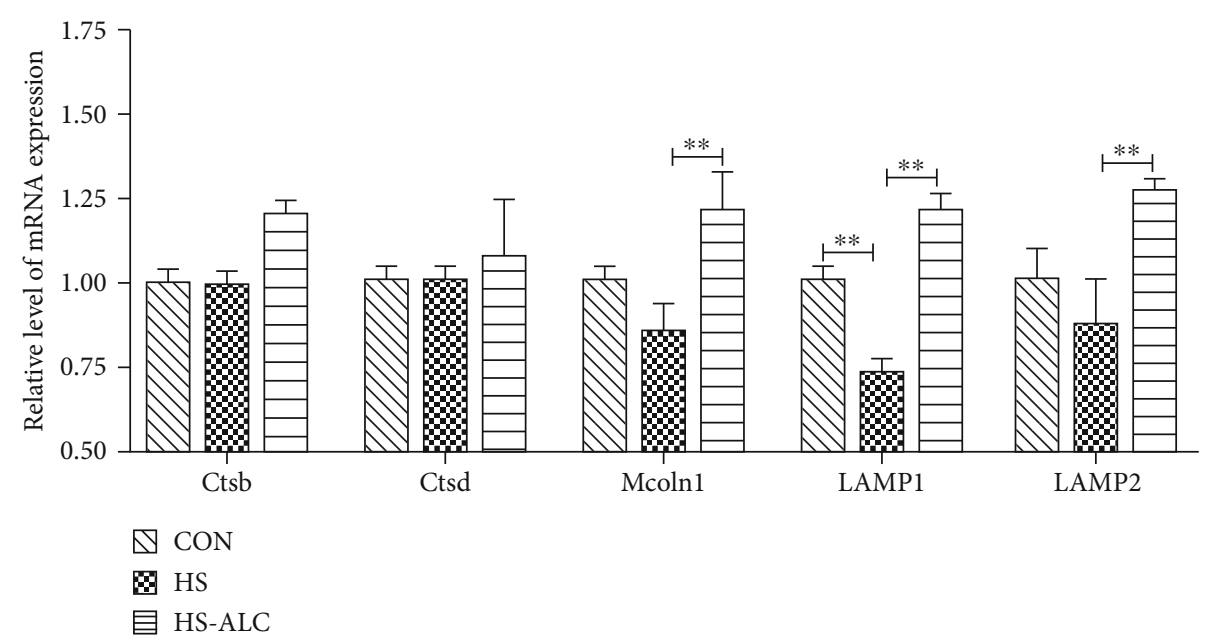

(1)

FIGURE 3: Effect of ALC on heat stress-induced autophagy in GC-1 cells. (a) Detection of autophagosomes using monodansylcadaverine (MDC) staining. (b) Percentages of MDC-positive cells. (c) mRNA levels of Atg5, Beclin1, and LC3II in GC-1 cells. (d, e) Western blot analysis of LC3II/LC3I protein expression in GC-1 cells. ( $\mathrm{f}, \mathrm{g}$ ) Western blot analysis of Beclin1 protein expression in GC-1 cells. (h) The immunofluorescence of LC3II in GC-1 cells. (i) The relative AOD of the LC3II positive cells. (j, k) Western blot analysis of p62 protein expression in GC-1 cells. (l) The mRNA levels of lysosomal biogenesis genes were determined using RT-PCR. The data are presented as the mean $\pm \mathrm{SD}, n=3,{ }^{*} P<0.05,{ }^{* *} P<0.01$. CON: control group; HS: recover at $6 \mathrm{~h}$; HS-ALC: recover at $6 \mathrm{~h}$ with ALCAR.

to the HS group $(P<0.05)$ (Figures 3(a) and 3(b)). What is more, heat exposure significantly increased mRNA levels of Atg5, Beclin1, and LC3II in GC-1 cells $(P<0.01)$ (Figure 3(c)). However, ALC treatment further increased the expression of Atg5, Beclin1, and LC3II mRNA in heat treatment cells $(P<0.05)$ (Figure 3(c)). Six hours after heat stress, we found that the protein levels of Beclin1 and LC3II/LC3I were remarkedly higher compared to the CON group $(P<0.01)$ (Figures $3(\mathrm{e})-3(\mathrm{~g}))$. Immunofluorescence data illustrated that the levels of LC3II were notably increased in the HS-ALC group compared to the CON group $(P<0.01)$, whereas the levels of LC3II were markedly increased in the HS-ALC group compared to the HS group $(P<0.05)$ (Figures $3(\mathrm{~h})$ and $3(\mathrm{i}))$. Furthermore, the protein levels of p62 markedly upregulated in the HS group compared to the CON group and the HS-ALC group $(P<0.05)$ (Figures $3(\mathrm{j})$ and $3(\mathrm{k})$ ). The mRNA levels of LAMP1 were markedly decreased in the HS group compared to the Con group $(P<0.01)$ (Figure 3(1)). Additionally, the expressions of Mcoln1, LAMP1, and LAMP2 were significantly increased in the HS-ALC group compared to the HS group $(P<0.01)$ (Figure 3(1)).

3.4. Effect of ALC on Apoptosis in GC-1. As shown in Figures 4(a) and 4(b), the rate of apoptotic cells was remarkably decreased in the CON group and the HS-ALC group compared to the HS group $(P<0.05)$. Additionally, the mRNA and protein levels of Bcl-2, Bax, caspase 3 were significantly increased in the HS group compared to the CON group $(P<0.05)$ (Figures $4(\mathrm{c})-4(\mathrm{~g}))$. The addition of ALC significantly upregulated the mRNA and protein levels of Bcl-2 and downregulated the mRNA and protein levels of caspase3 $(P<0.05)$ (Figures $4(\mathrm{c})-4(\mathrm{~g}))$. Furthermore, the protein levels of $\mathrm{Bcl}-2 / \mathrm{Bax}$ were significantly increased in the HS group compared to the CON group $(P<0.05)$ (Figures 4(d) and 4(h)).

\section{Discussion}

With global warming, all living beings are facing the adverse effects of it, and especially, the male animals are facing enormous challenges in reproduction. Usually, the harmful effect of heat stress on spermiogenesis in the mammal is mainly through inducing oxidative damage, disturbing the mitochondrial function, and increasing cell mortality [23]. Several studies have demonstrated the antioxidative and mitochondrial membrane stabilizing properties of $\operatorname{ALC}[17,24]$. However, little evidence is accessible about the role of ALC in promoting spermatogonia recovery from the damage of heat stress. In this study, we found that ALC attenuated HSinduced viability and apoptosis by reducing oxidative stress and upregulating autophagy after heat stress.

To investigate the protective role of ALC, cell viability and LDH leakage were conducted. Previous research has shown that the increased LDH content in cell supernatant is due to the increased cell membrane permeability under heat stress [25]. In this study, we also found the same phenomenon, the ascent of the concentration of LDH in the supernatant after heat stress. However, the LDH concentration decreased significantly when ALC was added to the cell medium before heat stress. At the same time, ALC treatment also increases cell viability. These findings show that ALC could protect the integrity of the GC-1 cell membrane to accelerate the recovery of GC-1 cells after thermal stress. Furthermore, we investigated the effect of the heat stress and ALC treatment on antioxidation against of GC-1 cells. The activities of intracellular 

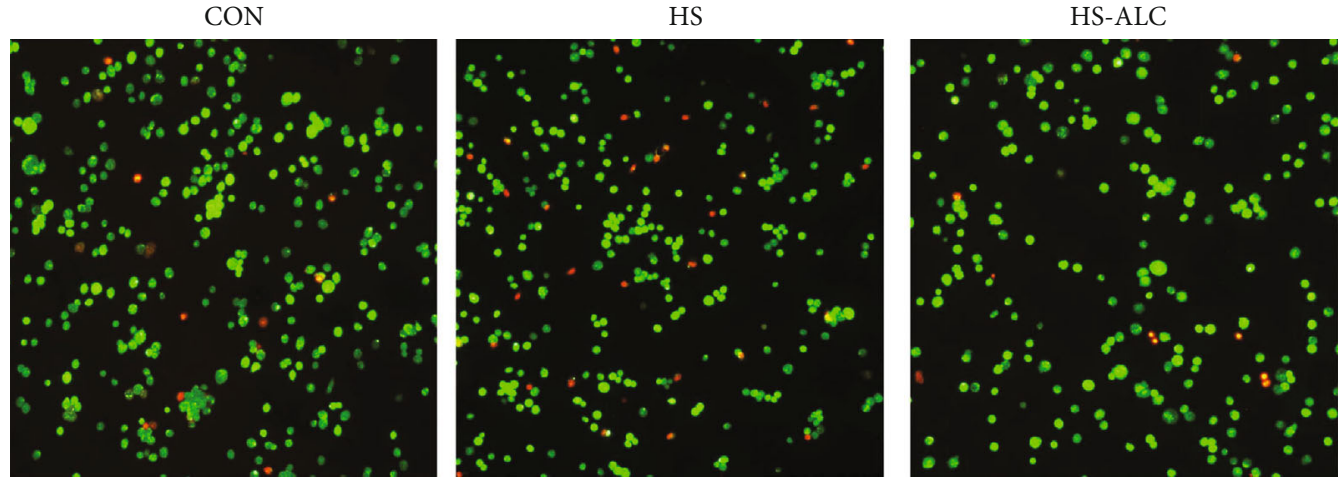

(a)
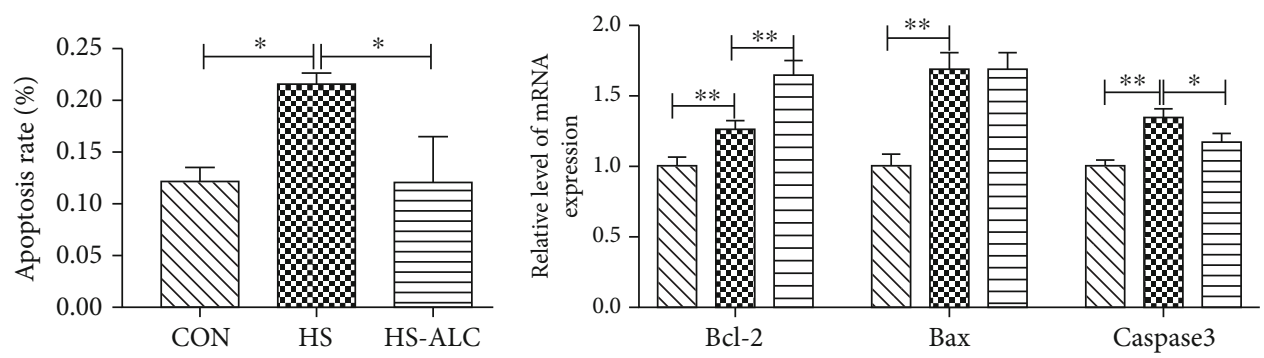

$\triangle \mathrm{CON}$

B $\mathrm{HS}$

目 HS-ALC

(b)

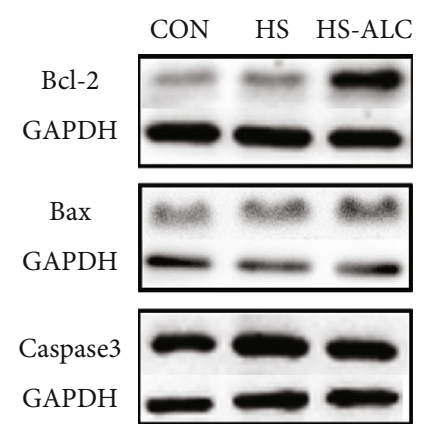

(d)

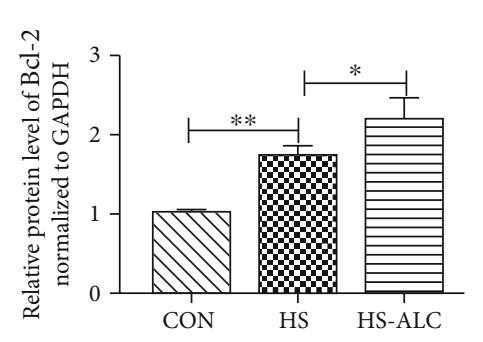

(e)

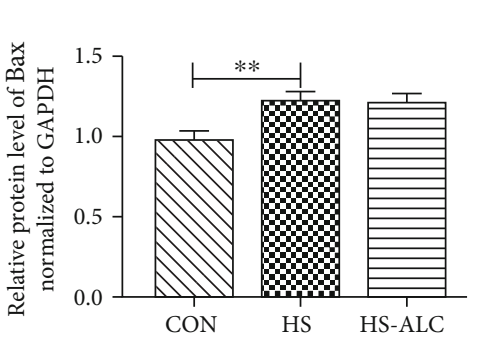

(f) (c)

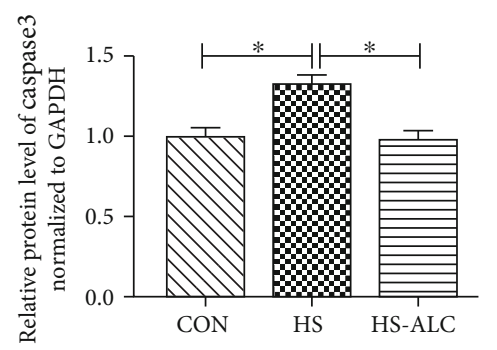

(g)

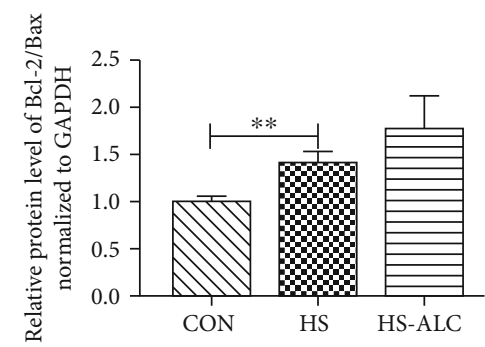

(h)

Figure 4: Effect of ALC on heat stress-induced apoptosis in GC-1 cells. (a) The cells were labeled with AO/EB and then analyzed by fluorescence microscope. (b) Data were expressed as the percent of apoptotic cells obtained from the histogram statistics all quantitative. (c) The mRNA levels of Bcl-2, Bax, and caspase3. (d) Immunoblot analysis of apoptosis-related proteins. (e, f) The protein levels of Bcl-2, Bax, caspase3, and Bcl-2/Bax. The data are presented as the mean $\pm \mathrm{SD}, n=3,{ }^{*} P<0.05,{ }^{*} P<0.01$. CON: control group; HS: recover at $6 \mathrm{~h}$; HS-ALC: recover at $6 \mathrm{~h}$ with ALCAR.

oxidation and antioxidant enzyme reflect the antioxidant capacity of the cells. CAT is considered an essential enzyme in the process of ROS elimination by decomposing hydrogen peroxide to $\mathrm{H}_{2} \mathrm{O}$ and $\mathrm{O}_{2}$ [26]. MDA is the final product of lipid peroxidation in cells and is considered a sign of oxidative stress [27]. T-AOC indicates the oxidation resistance capacity of the cell. In the present study, CAT, MDA, and T-AOC levels were markedly increased in GC-1 cells after recovered for $6 \mathrm{~h}$. So we speculated that GC-1 cells promoted cell recovery by increasing the activity of antioxidant enzymes (CAT and T- 
AOC) after heat stress. Nevertheless, the elevated activity of the antioxidant enzyme was not enough to against effects from heat; MDA was largely accumulated in cell treatment of heat. These finding indicated that GC-1 cells were severely oxidatively damaged, and oxidative stress may play an essential role in heat stress-induced damage. A recent in vivo study showed that ALC treatment significantly suppressed the oxidative damage induced by $\mathrm{NaAsO}_{2}$ through maintaining oxidantantioxidant balance [17]. In this research, we found that ALC has a similar protective function on the GC- 1 cells under heat stress. ALC treatment decreased LDH, MDA, and CAT levels in GC-1 cells after heat stress.

When cells are stimulated or damaged, many changes will take place in mitochondrial biological function, such as the change of mitochondrial membrane permeability and the loss of mitochondrial membrane potential [22]. In this study, we found that HS causes a loss of MMP by $35 \%$ compared to the control group. Conversely, ALC treatment reduced HSinduced decrease in MMP. The loss of MMP is closely related to programmed cell death and mitochondrial apoptosis [28]. So we examined the expression of apoptosis-related genes. The Bcl-2 protein family plays a decisive role in the regulation of apoptosis, which may either inhibit (Bcl-2) or promote apoptosis (Bax). Cell apoptosis can be evaluated by the ratio of Bcl2 and Bax [29]. In response to various cellular stresses, the apoptotic factors of the Bcl-2 family change the permeability of the mitochondrial outer membrane and release apoptotic factors into the cytosol [30]. Caspase9 is then activated by these proapoptotic factors [31]. Finally, active caspase9 cleaves and activates the executioner caspase 3 . In the present study, the apoptotic rate was increased in the HS group compared to the CON group. Moreover, the expression of proapoptotic genes and protein (Bax and caspase3) increased significantly in the HS group compared to the CON group. However, we also found that heat stress increases the expression of apoptosis suppressor gene and protein $\mathrm{Bcl}-2$. In previous studies, Bcl-2 mRNA and protein levels in testes and mouse granulosa cells were also increased after heat stress, but they did not have enough direct evidence to clarify the reason for the rise of Bcl2 in heat-stressed cells [32-34]. One of these studies suggested that the increasing level of Bcl-2 mRNA expression might be the result of heat shock proteins, which was a self-protection mechanism in granulosa cells [34]. Therefore, we also speculated that the recovery of GC- 1 cells after heat stress might be related to the upregulation of antiapoptotic gene Bcl-2. Furthermore, treatment with ALC decreased the apoptotic rate and expression of caspase $3 \mathrm{mRNA}$ and protein levels and increased the expression of Bcl-2 mRNA and protein levels in GC-1 cells. Based on these results, it seems possible that ALC might promote the recovery of GC-1 cells after heat stress through increasing Bcl-2 expression and decreasing caspase 3 expression.

Autophagy, or type II programmed cell death, is a cellular process to remove the damaged organelles and protein aggregates [35]. During apoptosis, the stimulation of autophagy can be either a protective mechanism or a process that contributes to cell death [36]. Heat stress has become an important issue in stimulating autophagy [37]. Acidic vesicles occur during autophagy, so MDC staining is often used to monitor autophagy. In this study, the results of the MDC staining showed that the number of autophagosomes was markedly increased after cell recovery for $6 \mathrm{~h}$ and the number of autophagosomes was further increased while the cells treated with ALC. In addition, there are several genes that are closely related to the process of autophagy. Autophagyrelated gene $5(\operatorname{Atg} 5)$ and beclin1 are primary for the process of autophagy. Beclin1 is a platform by binding several cofactors to assemble the class III phosphatidylinositol 3-kinase (PI3K) complex during autophagosome formation [38]. When phagophore is elongating, the Atg5-Atg12 conjugating system combines with Atg16L1 to form Atg5-Atg12-Atg16L1. Then, this complex further prolongs the phagophore and is required for the LC3 lipidation [39]. When autophagy is triggered, cytosolic LC3 is cleaved to form LC3I. Then, LC3I binds to phosphatidylethanolamine to become LC3II which is recruited to the membrane of the autophagosome. The ratio of LC3II to LC3I is used to assess autophagy levels [40]. Then, the p62 bind to LC3 and deliver specific organelles and protein aggregates to autophagosomes for degradation [41]. Finally, the polyubiquitin-binding protein $\mathrm{p} 62$ is degraded by autophagy [42]. Here, we found that the expression of Atg5, beclin1, and LC3II mRNA levels and the AOD of LC3II were increased, but the protein levels of p62 were upregulated in the HS group compared to the CON group. These results indicated that increased autophagosome formation was not caused by autophagy but rather by a decrease in autophagosome clearance, as reflected by accumulation of p62. The significant decrease of the lysosomal transmembrane proteinsrelated genes of LAMP1 also proved this point. However, ALC treatment effectively promoted the mRNA and protein levels of beclin1 and LC3II, increased the AOD of LC3II and expression of lysosomal biogenesis-related genes (Mcoln1, LAMP1, and LAMP2) in GC-1 cells but decreased the protein levels of $\mathrm{p} 62$ under heat stress. These data suggested that the ALC could promote the recovery of GC1 cells after heat stress by increasing the level of autophagy. Autophagy and apoptosis are regulated by the same regulatory genes, so there is a crosstalk between them [43]. Bcl-2 is one of the regulatory genes. Bcl-2 can bind to Beclin1, prevent the association of Beclin-1 with PI3K complex, and inhibit autophagy [44]. However, the phosphorylated Bcl-2 loses its ability it inhibits autophagy [45]. In this study, we found the Bcl-2 and Beclin-1 were increased, but the levels of autophagy were increased in the HS-ALC group compared to the HS group. So we speculated that phosphorylated Bcl-2 may be involved in the regulation of autophagy in GC-1 cell treatment with ALC after heat stress. More detailed studies of the phosphorylation of $\mathrm{Bcl}-2$ will be needed to obtain a better understanding of the crosstalk between autophagy and apoptosis.

In summary, our research provides the first evidence that ALC can promote GC-1 cells recovery after heat stress by reducing oxidative stress and upregulating autophagy.

\section{Data Availability}

The data used to support the findings of this study are available from the corresponding author upon request. 


\section{Conflicts of Interest}

The authors declared that they have no conflicts of interest in this work.

\section{Acknowledgments}

The funding for this program is provided by the National Key R\&D Program of China (No. 2017YFD0502200 and 2016YFD0501205) and the National Science Foundation for Yong Scientists of China (No. 31502131).

\section{References}

[1] Z. Y. Niu, F. Z. Liu, Q. L. Yan, and W. C. Li, "Effects of different levels of vitamin $\mathrm{E}$ on growth performance and immune responses of broilers under heat stress," Poultry Science, vol. 88, no. 10, pp. 2101-2107, 2009.

[2] C. G. Crandall and T. E. Wilson, "Human cardiovascular responses to passive heat stress," Comprehensive Physiology, vol. 5, no. 1, pp. 17-43, 2015.

[3] R. Mieusset and L. Bujan, "Testicular heating and its possible contributions to male infertility: a review," International Journal of Andrology, vol. 18, no. 4, pp. 169-184, 1995.

[4] D. Durairajanayagam, A. Agarwal, and C. Ong, "Causes, effects and molecular mechanisms of testicular heat stress," Reproductive Biomedicine Online, vol. 30, no. 1, pp. 14-27, 2015.

[5] E. Carlsen, A. M. Andersson, J. H. Petersen, and N. E. Skakkebaek, "History of febrile illness and variation in semen quality," Human Reproduction, vol. 18, no. 10, pp. 2089-2092, 2003.

[6] Y. H. Lue, A. P. Hikim, R. S. Swerdloff et al., "Single exposure to heat induces stage-specific germ cell apoptosis in rats: role of intratesticular testosterone on stage specificity," Endocrinology, vol. 140, no. 4, pp. 1709-1717, 1999.

[7] B. Zhu, S. K. Walker, H. Oakey, B. P. Setchell, and S. Maddocks, "Effect of paternal heat stress on the development in vitro of preimplantation embryos in the mouse," Andrologia, vol. 36, no. 6, pp. 384-394, 2004.

[8] M. Kanter and C. Aktas, "Effects of scrotal hyperthermia on Leydig cells in long-term: a histological, immunohistochemical and ultrastructural study in rats," Journal of Molecular Histology, vol. 40, no. 2, pp. 123-130, 2009.

[9] C. Paul, S. Teng, and P. T. K. Saunders, "A single, mild, transient scrotal heat stress causes hypoxia and oxidative stress in mouse testes, which induces germ cell death," Biology of Reproduction, vol. 80, no. 5, pp. 913-919, 2009.

[10] D. J. McLean, L. D. Russell, and M. D. Griswold, "Biological activity and enrichment of spermatogonial stem cells in vitamin A-deficient and hyperthermia-exposed testes from mice based on colonization following germ cell transplantation," Biology of Reproduction, vol. 66, no. 5, pp. 1374-1379, 2002.

[11] A. Coto-Montes, J. A. Boga, S. Rosales-Corral, L. FuentesBroto, D. X. Tan, and R. J. Reiter, "Role of melatonin in the regulation of autophagy and mitophagy: a review," Molecular and Cellular Endocrinology, vol. 361, no. 1-2, pp. 12-23, 2012.

[12] B. S. Song, S. B. Yoon, J. S. Kim et al., "Induction of autophagy promotes preattachment development of bovine embryos by reducing endoplasmic reticulum stress," Biology of reproduction, vol. 87, pp. 1-11, 2012.
[13] M. Q. Zhang, M. Jiang, Y. Bi, H. Zhu, Z. M. Zhou, and J. H. Sha, "Autophagy and apoptosis act as partners to induce germ cell death after heat stress in mice," Plos One, vol. 7, 2012.

[14] Z. Li, Y. Li, X. Zhou, P. Dai, and C. Li, “Autophagy involved in the activation of the Nrf2-antioxidant system in testes of heatexposed mice," Journal of Thermal Biology, vol. 71, pp. 142152, 2018.

[15] X. Li, C. Zhang, X. Zhang et al., “An acetyl-L-carnitine switch on mitochondrial dysfunction and rescue in the metabolomics study on aluminum oxide nanoparticles," Particle and Fibre Toxicology, vol. 13, p. 4, 2016.

[16] G. Peluso, O. Petillo, A. Barbarisi et al., "Carnitine protects the molecular chaperone activity of lens alpha-crystallin and decreases the post-translational protein modifications induced by oxidative stress," The FASEB Journal, vol. 15, no. 9, pp. 1604-1606, 2001.

[17] V. Bodaghi-Namileh, M. R. Sepand, A. Omidi et al., "Acetyl-1carnitine attenuates arsenic-induced liver injury by abrogation of mitochondrial dysfunction, inflammation, and apoptosis in rats," Environmental Toxicology and Pharmacology, vol. 58, pp. 11-20, 2018.

[18] H. M. Abdul and D. A. Butterfield, "Involvement of PI3K/PKG/ERK1/2 signaling pathways in cortical neurons to trigger protection by cotreatment of acetyl-L-carnitine and $\alpha$-lipoic acid against HNE-mediated oxidative stress and neurotoxicity: implications for Alzheimer's disease," Free Radical Biology \& Medicine, vol. 42, no. 3, pp. 371-384, 2007.

[19] L. Nicassio, F. Fracasso, G. Sirago et al., "Dietary supplementation with acetyl-l-carnitine counteracts age-related alterations of mitochondrial biogenesis, dynamics and antioxidant defenses in brain of old rats," Experimental Gerontology, vol. 98, pp. 99-109, 2017.

[20] S. Micic, N. Lalic, D. Djordjevic et al., "Double-blind, randomised, placebo-controlled trial on the effect of L-carnitine and L-acetylcarnitine on sperm parameters in men with idiopathic oligoasthenozoospermia," Andrologia, vol. 51, no. 6, p. e13267, 2019.

[21] G. M. Busetto, A. Agarwal, A. Virmani et al., "Effect of metabolic and antioxidant supplementation on sperm parameters in oligo-astheno-teratozoospermia, with and without varicocele: A double-blind placebo-controlled study," Andrologia, vol. 50, 2018.

[22] F. Yang, J. Liao, R. Pei et al., "Autophagy attenuates copperinduced mitochondrial dysfunction by regulating oxidative stress in chicken hepatocytes," Chemosphere, vol. 204, pp. 36-43, 2018.

[23] M. B. Rahman, K. Schellander, N. L. Luceno, and A. Van Soom, "Heat stress responses in spermatozoa: mechanisms and consequences for cattle fertility," Theriogenology, vol. 113, pp. 102-112, 2018.

[24] S. Singh, A. Mishra, and S. Shukla, "ALCAR exerts neuroprotective and pro-neurogenic effects by inhibition of glial activation and oxidative stress via activation of the $\mathrm{Wnt} / \beta$-catenin signaling in parkinsonian rats," Molecular Neurobiology, vol. 53, no. 7, pp. 4286-4301, 2016.

[25] Y. F. Cheng, J. R. Sun, H. B. Chen et al., "Association of Hsp60 expression with damage to rat myocardial cells exposed to heat stress in vivo and in vitro," Genetics and Molecular Research, vol. 13, no. 4, pp. 9371-9381, 2014.

[26] W. S. Habashy, M. C. Milfort, R. Rekaya, and S. E. Aggrey, "Cellular antioxidant enzyme activity and biomarkers for 
oxidative stress are affected by heat stress," International Journal of Biometeorology, vol. 63, no. 12, pp. 1569-1584, 2019.

[27] D. Tsikas, "Assessment of lipid peroxidation by measuring malondialdehyde (MDA) and relatives in biological samples: analytical and biological challenges," Analytical Biochemistry, vol. 524, pp. 13-30, 2017.

[28] F. Yang, R. N. Pei, Z. W. Zhang et al., "Copper induces oxidative stress and apoptosis through mitochondria-mediated pathway in chicken hepatocytes," Toxicology In Vitro, vol. 54, pp. 310-316, 2019.

[29] X. X. Yuan, B. Zhang, L. L. Li et al., "Effects of soybean isoflavones on reproductive parameters in Chinese mini-pig boars," Journal of Animal Science and Biotechnology, vol. 3, no. 1, p. 31, 2012.

[30] A. Ashkenazi and G. Salvesen, "Regulated cell death: signaling and mechanisms," Annual Review of Cell and Developmental Biology, vol. 30, no. 1, pp. 337-356, 2014.

[31] S. B. Bratton and G. S. Salvesen, "Regulation of the Apaf-1-caspase-9 apoptosome," Journal of Cell Science, vol. 123, no. 19, pp. 3209-3214, 2010.

[32] C. M. Yamamoto, A. P. Sinha Hikim, P. N. Huynh et al., "Redistribution of Bax is an early step in an apoptotic pathway leading to germ cell death in rats, triggered by mild testicular hyperthermia," Biology of Reproduction, vol. 63, no. 6, pp. 1683-1690, 2000.

[33] Z. H. Zhang, X. Jin, X. S. Zhang et al., "Bcl-2 and Bax are involved in experimental cryptorchidism-induced testicular germ cell apoptosis in rhesus monkey," Contraception, vol. 68, no. 4, pp. 297-301, 2003.

[34] M. Luo, L. Li, C. Xiao, Y. Sun, and G. L. Wang, "Heat stress impairs mice granulosa cell function by diminishing steroids production and inducing apoptosis," Molecular and Cellular Biochemistry, vol. 412, no. 1-2, pp. 81-90, 2016.

[35] P. Ravanan, I. F. Srikumar, and P. Talwar, "Autophagy: the spotlight for cellular stress responses," Life Sciences, vol. 188, pp. 53-67, 2017.

[36] L. A. Booth, S. Tavallai, H. A. Hamed, N. Cruickshanks, and P. Dent, "The role of cell signalling in the crosstalk between autophagy and apoptosis," Cellular Signalling, vol. 26, no. 3, pp. 549-555, 2014.

[37] S. H. Wang, C. Y. Cheng, C. J. Chen et al., "Acute heat stress changes protein expression in the testes of a broiler-type strain of Taiwan country chickens," Animal Biotechnology, vol. 30, no. 2, pp. 129-145, 2019.

[38] Q. M. Sun, W. L. Fan, K. L. Chen, X. J. Ding, S. Chen, and Q. Zhong, "Identification of Barkor as a mammalian autophagy-specific factor for Beclin 1 and class III phosphatidylinositol 3-kinase," Proceedings of the National Academy of Sciences, vol. 105, no. 49, pp. 19211-19216, 2008.

[39] C. Otomo, Z. Metlagel, G. Takaesu, and T. Otomo, "Structure of the human ATG12 ATG5 conjugate required for LC3 lipidation in autophagy," Nature Structural \& Molecular Biology, vol. 20, no. 1, pp. 59-66, 2013.

[40] Y. Kabeya, N. Mizushima, T. Ueno et al., "LC3, a mammalian homolog of yeast Apg8p, is localized in autophagosome membranes after processing (vol 19, pg 5720, 2000)," The EMBO Journal, vol. 22, pp. 4577-4577, 2003.

[41] T. Lamark, S. Svenning, and T. Johansen, "Regulation of selective autophagy: the p62/SQSTM1 paradigm," Essays in Biochemistry, vol. 61, no. 6, pp. 609-624, 2017.
[42] G. Zaffagnini, A. Savova, A. Danieli et al., "p62 filaments capture and present ubiquitinated cargos for autophagy," The EMBO journal, vol. 37, 2018.

[43] K. Wang, "Autophagy and apoptosis in liver injury," Cell cycle, vol. 14, pp. 1631-1642, 2015.

[44] G. Robert, C. Gastaldi, A. Puissant et al., "The anti-apoptotic Bcl-B protein inhibits BECN1-dependent autophagic cell death," Autophagy, vol. 8, pp. 637-649, 2014.

[45] Y. Wei, S. Pattingre, S. Sinha, M. Bassik, and B. Levine, "JNK1mediated phosphorylation of Bcl-2 regulates starvationinduced autophagy," Molecular Cell, vol. 30, no. 6, pp. 678688, 2008. 